exacerbation of spinal cord injuries. Application of rigid cervical collar may reduce cervical spine movements, but it hinders tracheal intubation with standard laryngoscope. It significantly reduces the mouth opening, rendering laryngoscopy difficult and also lifts up the chin and tips the larynx anteriorly. The aim of this study is to compare the hemodynamic responses to fibreoptic bronchoscope and McCoy laryngoscope in patients undergoing elective surgery under general anesthesia with rigid cervical collar simulating cervical spine immobilization in the situation of cervical trauma. Materials and Methods: Thirty two patients in age range 20-50 years, of ASA I - $\Pi$, and of either sex undergoing elective surgery under general anesthesia were randomly allocated into each group. There were two groups according to the technique used for intubation: Group A (Flexible Fibreoptic Bronchoscope) and group B (McCoy Laryngoscope). Systolic Blood Pressure (SBP), Diastolic Blood Pressure (DBP), Mean arterial blood pressure (MAP) and heart rate (HR) were recorded at baseline, intra-operatively, immediately before and after induction, and immediately after intubation. Thereafter, every minute for the next five minutes. Demographic data is presented as numbers and intergroup comparison of these was done by Chi square test. The value of $P<0.05$ was considered statistically significant. Quantitative data is presented as mean values and standard deviation. Intergroup comparison of quantitative data was done by parametric test (unpaired $t$-test) and probability was considered to be significant if less than $<0.05$. Results: The demographic data is comparable. Due to intubation response, heart rate and blood pressure increased significantly $(P<0.05)$ above preoperative values in McCoy group as compared to fibreoptic group. Conclusion: We suggest that the flexible fibreoptic bronchoscope is an effective and better method of intubation in situation like traumatic cervical spine injury and provides stable hemodynamics.

\section{Attenuation of hemodynamic pressor response to laryngoscopy and intubation with single pre-induction dose of dexmedetomidine in patients undergoing neurosurgery and neurointervention}

\section{Neeta Karmarkar, Rajashree Gandhe}

Department of Anaesthesia, Kokilaben Dhirubhai Ambani Hospital, Mumbai, Maharashtra, India

Background: The aim of the study was to evaluate the effect of a single pre induction dose of dexmedetomidine on pressor response to laryngoscopy and intubation on patients undergoing neurosurgery and neurointervention and to assess the incidence of adverse effects of dexmedetomidine. Materials and Methods: After obtaining the approval of the hospital ethics committee an observational study was conducted on 100 ASA grade 1 and 2 patients. Written, informed valid consent was obtained from all patients. Inj. Dexmedetomidine was started in an infusion at the rate of $1 \mathrm{mcg} / \mathrm{kg}$ for ten minutes before induction. Heart rate and blood pressure were then recorded at specific intervals before and after intubation. Results: Sixty patients were enrolled for the study. The baseline heart rate was 77.15 (SD 14.49) beats per minute (bpm). One minute after intubation mean heart rate was 121.44 (SD 22.34) bpm. The baseline mean arterial pressure was 109.29 (SD 14.58) $\mathrm{mm} \mathrm{Hg}$. One minute after intubation mean value was 95.06 (SD 17.89) $\mathrm{mm} \mathrm{Hg}(P<0.001)$. The difference was significant in all parameters at the said intervals as compared to the baseline. No adverse effects were reported. Conclusion: Dexmedetomidine is a very useful drug as a premedicant @1 mcg/ $\mathrm{kg}$ in an infusion for ten minutes to attenuate the sympathetic response to laryngoscopy and intubation.

\section{A prospective randomized control evaluation of desflurane and propofol for emergence from anesthesia following resection of cerebellopontine angle tumors}

\section{Sohan Lal Solanki, Manohar Bhat, Hemant Bhagat ${ }^{1}$, Ishwar Bhukal ${ }^{1}$}

Departments of Anaesthesia, Critical Care and Pain, Tata Memorial Hospital, Mumbai, Maharashtra, ${ }^{1}$ Anaesthesia and Intensive Care, PGIMER, Chandigarh, India

Background:Desflurane and propofol are commonly used anesthetic agent in neurosurgical patients. Though these agents have been commonly used in patients undergoing supratentorial neurosurgery, there is paucity of data evaluating its use in surgery for cerebellopontine $(\mathrm{CP})$ angle tumors. The primary aim was to evaluate the time to emergence from anesthesia with use of desflurane when compared to propofol. The intraoperative hemodynamics, brain relaxation and vasomotor response during tumor resection were the secondary outcomes. Materials and Methods: The present study was a prospective randomized trial conducted on thirty adult patients undergoing $\mathrm{CP}$ angle tumor resection. Anesthesia was induced with morphine $0.1 \mathrm{mg} / \mathrm{kg}$ and thiopentone $4-6 \mathrm{mg} / \mathrm{kg}$ while muscle relaxation was achieved with vecuronium. Anesthesia was maintained with desflurane or propofol as per the randomization along with nitrous oxide (NO) to maintain a state 2 entropy value of 40-60. Desflurane or propofol were switched off after completion of skin sutures while NO was switched off following removal of skull pins. 